ADVERSE DRUG REACTION

\title{
Colonic ulcers in propylthiouracil induced vasculitis with secondary antiphospholipid syndrome
}

\author{
P D Gaburri, J M F Chebli, Â Attalla, C M N Pereira, H L Bonfante, E V Martins Junior, A K Gaburri
}

Postgrad Med J 2005;81:338-340. doi: 10.1136/pgmj.2004.026104

\begin{abstract}
A 48 year old white woman was admitted to the hospital because of several bouts of migratory polyarthritis, weight loss, fever, and abdominal pain over a period of 15 months. She had been taking propylthiouracil $100 \mathrm{mg}$ daily for three years for hyperthyroidism treatment. A test for antineutrophil cytoplasmic autoantibodies (ANCA) was positive with a perinuclear pattern of staining. Antiphospholipid antibodies were also detected. Colonoscopy showed several ulcers on intestinal mucosa and the biopsy specimen showed intense microscopic vasculitis. The patient is well after methylprednisolone pulse therapy and eight months of oral azathioprine. A surveillance colonoscopy showed complete healing of intestinal ulcers. No recurrence of symptoms has occurred and autoantibodies are negative, 10 months after treatment finished. The sequence of events suggests a propylthiouracil induced vasculitis p-ANCA positive and an antiphospholipid syndrome. This is the first report of colonic ulcers diagnosed and successfully treated in such circumstances.
\end{abstract}

$\mathrm{V}$ asculitides affect several organs or an organ system and always have to be regarded as systemic diseases. Their gastrointestinal manifestations are a continuous challenge for gastroenterologists. ${ }^{1}$ The clinical picture of gastrointestinal vasculitides can be acute or chronic. More than $90 \%$ of chronic vascular diseases of the gastrointestinal tract are attributable to arteriosclerosis while less than $10 \%$ are caused by chronic intestinal vasculitides. They can be primary or secondary. The first group includes those involving large, medium, or small size vessels. The secondary group comprises those as a consequence of rheumatic and autoimmune diseases as well as infections, drugs, toxic substances, and paraneoplastics. Drugs related to occurrence of vasculitides are antibiotics, non-steroidal antirheumatic drugs, chloroquine, D-penicillamine, gold, carbimazole, angiotensin converting enzyme inhibitors, cytotoxic drugs (chemotherapy), and dextrane. ${ }^{1}$ Propylthiouracil (PTU) can induce ANCA positive vasculitides with polyclonal autoantibodies. ${ }^{5}$ Occurrence of PTU induced ANCA positive vasculitides has been reported, but there are few references to gastrointestinal lesions occurrence..$^{2-4}$ Antiphospholipid syndrome denotes the clinical association between antiphospholipid antibodies and a syndrome of hypercoagulability but the criteria for diagnosis are an ongoing process. Antiphospholipid antibodies are a family of autoantibodies that exhibit a broad range of target specificities and affinities, all recognising various combinations of phospholipids, phospholipids binding proteins, or both. ${ }^{8}$

\section{CASE REPORT}

A 48 year old white woman was admitted to the hospital because of severe abdominal pain, weight loss, bouts of migratory polyarthritis, and fever during the past 15 months. She was taking propylthiouracil $100 \mathrm{mg}$ a day over a period of three years for hyperthyroidism. The symptoms had worsened in the past two months and an intense deterioration of general physical state occurred. She needed frequent use of opioids orally in the past two weeks to relieve abdominal pain. There was no blood or mucus in the stools. On admission she was pale-haemoglobin concentration $9.4 \mathrm{~g} \%$-and her temperature was $38^{\circ} \mathrm{C}$. Blood pressure was 120/80 $\mathrm{mm} \mathrm{Hg}$ and a painful hepatomegaly was noticed. The spleen was felt $7 \mathrm{~cm}$ below the left costal margin and an intense tenderness on the right lower quadrant of abdomen was remarkable on physical examination. An abdominal Doppler scan did not show portal tract or hepatic vein thrombosis. In addition, an abdominal computed tomogram did not show the presence of mesenteric thrombus, although thickness of distal ileum, caecum, and right colon wall was noticed. Neurological, musculoskeletal, ophthalmological, or gynaecological examination did not show abnormalities. Table 1 shows the results of the laboratory studies performed on admission. Blood culture and Widal's test were negative. An upper gastrointestinal endoscopy was normal. However, a colonoscopy showed several shallow ulcers from the caecum to splenic flexure and the biopsy samples from colonic ulcers displayed lymphocytes infiltration, fibrinoid necrosis of capillaries with fibrinous thrombus in their lumen (fig 1). A p-ANCA test and a cardiolipine antibody were positive and high level of antimyeloperoxidase antibody was detected. The diagnosis of a PTU induced vaculitides and antiphospholipid syndrome involving the gastrointestinal tract was assumed. The PTU administration was stopped. Venous thrombosis prophylaxis with low molecular weight heparin subcutaneously twice daily was started from admission and because of concern of antiphospholipid syndrome, warfarin $5 \mathrm{mg}$ daily was prescribed. Fever and intense abdominal pain persisted and she received a venous pulse therapy with $250 \mathrm{mg}$ of methylprednisolone twice daily for three days. A dramatic improvement occurred. She was given radioiodine therapy for hyperthyroidism and discharged receiving azathioprine $2 \mathrm{mg} / \mathrm{kg} /$ day for the following eight months. A follow up colonoscopy at this time showed complete healing of mucosal ulcers. The autoantibodies became negative including p-ANCA and she was free of symptoms 10 months after stopping immunossupression.

\section{DISCUSSION}

Autoimmune disorders can occur as serious complications of antithyroid drugs. Vasculitis can appear months after drug withdrawal. A high prevalence of ANCA and myeloperoxydase antibody can be found even without vasculitis, in patients receiving PTU. ${ }^{67}$ In our patient, indirect immunofluorescence

Abbreviations: ANCA, antineutrophil cytoplasmic autoantibody; PTU, propylthiouracil 

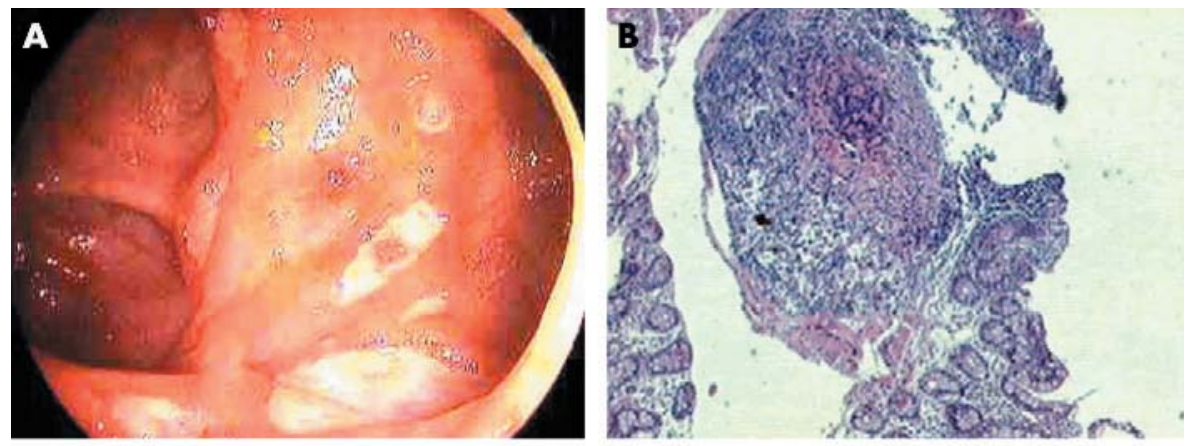

Figure 1 (A) Colonoscopy showing several ulcers in the caecum. Microscopy of ulcers biopsy with lymphocytes infiltration, necrosis, and thrombus in small size vessels lumen. ((B) haemotoxylin and eosin original magnification $\times 20$; $(C)$ and $(D)$ haemotoxylin and eosin original magnification $\times 40$ ).
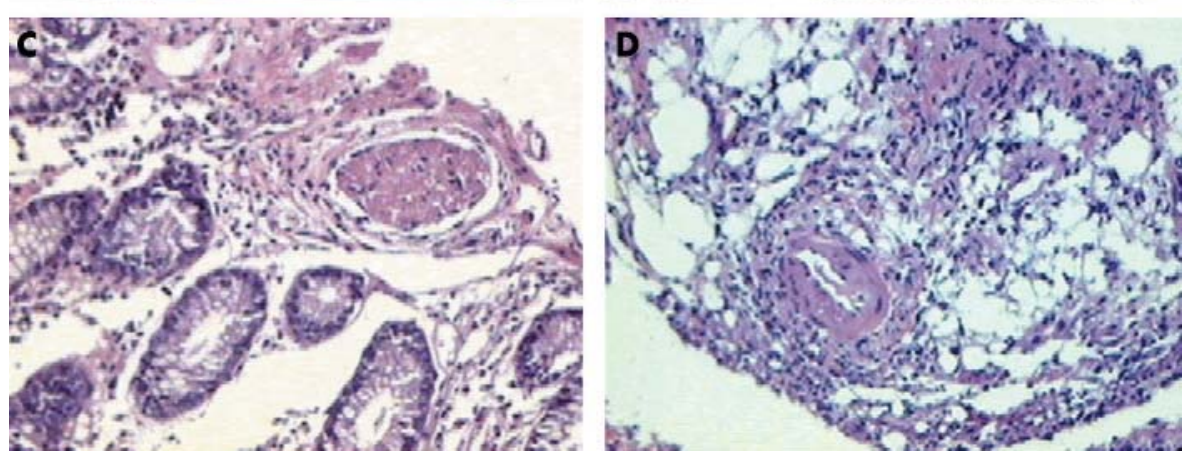

showed a perinuclear pattern staining of neutrophils (pANCA), which is associated with antimyeloperoxidase antibody and related to clinical vasculitis. This is the first report in the literature that describes endoscopic finding of ulcers in gastrointestinal tract in this sort of vasculitis. One fatal case of intestinal perforation has been described. ${ }^{2}$ Although PTU induced vasculitides can affect medium size vessels, some reported cases present only involvement of small size vessels without lung or kidney disease. This kind of vasculitis should be treated by urgent withdrawal of the drug, implementation of supportive measures, and immunosuppressive agents if necessary. ${ }^{4}$ In this patient receiving long term treatment with PTU, the histological pattern of microscopic vasculitis in colonic ulcers biopsy specimens, after the healing of the ulcers with the treatment used, allowed us to conclude that she suffered from a PTU induced ANCA positive vasculitis, with severe gastrointestinal involvement. Antiphospholipid syndrome was also considered

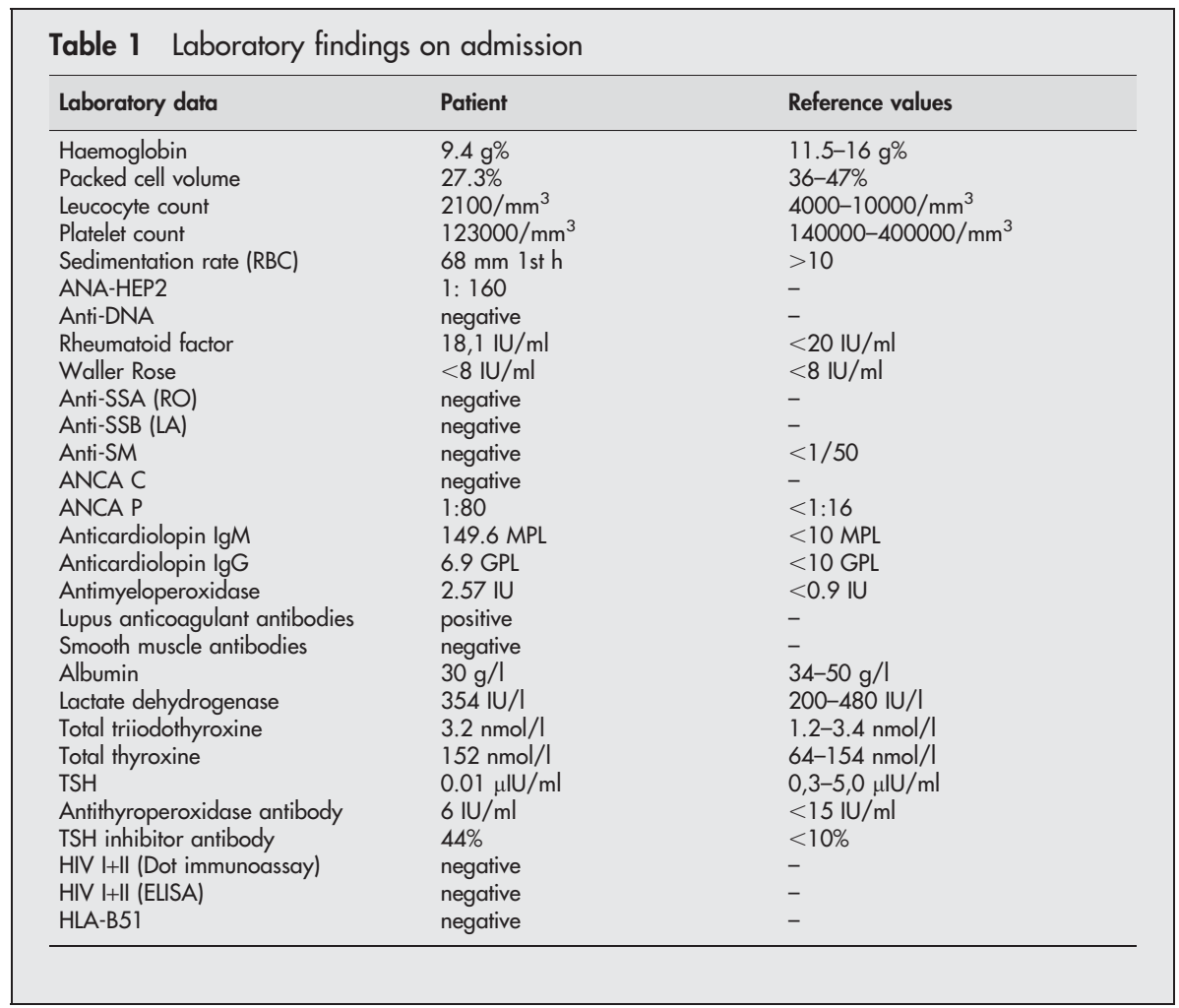


because of detection of high levels of anticardiolipin antibody IgM and lupus anticoagulant antibodies whose persistence occurred for more than six weeks associated with thrombosis. The most commonly detected antiphospholipid antibodies in antiphospholipid syndrome are lupus anticoagulant, anticardiolipin, and anti- $\beta_{2}$-glycoprotein I antibodies. Despite their name, lupus anticoagulant antibodies are associated with thromboembolic events rather than clinical bleeding. The anticardiolipin antibodies are strongly directed at an epitope on $\beta_{2}$-glycoprotein I and cause the development of anti- $\beta_{2}$-glycoprotein I antibodies that are strongly associated with thrombosis. ${ }^{8}$ Curiously, we could not precisely explain the reasons for liver and spleen enlargement in this case. Firstly, she was suspected to be suffering from Budd-Chiari syndrome. However, a Doppler scan of the portal tract and hepatic veins was normal and no signs of main hepatic vein thrombosis were found. We can speculate that liver and spleen enlargement could have been a consequence of the presence of hyaline thrombus in lobular terminal hepatic veins, because of fast remission after anticoagulation, although only a liver biopsy would be able to confirm that this hypothesis was correct. However, the patient's condition at that time did not allow us to do it.

In summary, several reports of PTU related vasculitides have been described but to the best of our knowledge this is the first case where colonic ulcers were diagnosed through colonoscopy and treated successfully. Withdrawal of PTU is usually enough to promote vasculitis remission, but corticosteroids or immunossupressive therapy may be necessary. No signs of kidney and skin damage were found in this patient, although commonly reported in PTU induced vasculitis.
Authors' affiliations

P D Gaburri, J M F Chebli, Â Attalla, Department of Medicine, Federal University of Juiz de Fora, Brazil

C M N Pereira, H L Bonfante, E V Martins Junior, A K Gaburri, Monte Sinai Hospital, Juiz de Fora, Brazil

Funding: none.

Conflicts of interest: none declared.

Correspondence to: Professor P D Gaburri, Department of Medicine, Federal University of Juiz de Fora, Rue São Sebastião 1050/301, Juiz de Fora, Brazil; pgaburri@medicina.ufif.br

Submitted 29 June 2004

Accepted 4 October 2004

\section{REFERENCES}

1 Müller-Ladner U. Vasculitides of the gastrointestinal tract. Best Practice and Research Clinical Gastroenterology 2001;15:59-82.

2 Reidy TJ, Upshaw JD, Chesney TM. Propylthiouracil-induced vasculitis: a fatal case. South Med J 1982;128:543-6.

3 Guo XH, Zhao MH, Gao Y, et al. Antineutrophil cytoplasmic antibody associated vasculitis induced by antithyroid agents. Zhonghua Yi Xue Za Zhi 2003;83:932-5.

4 Ligier S, Pham CD, Watters AK, et al. Purpura fulminans and anticardiolipin antibodies in a patient with Grave's disease. Scand J Rheumatol 2002;31:371-3.

5 Xu X, Zhao M, Zhang Y, et al. Clinicopathological characteristics of propylthiouracil-induced antineutrophil cytoplasmic antibodies-positive vasculitis and their target antigens: a report of 4 cases and literature review. Zhonghua Nei Ke Za Zhi 2002;41:404-7.

6 Sato $\mathrm{H}$, Hattori $M$, Fujieda $M$, et al. High prevalence of antineutrophil cytoplasmic antibody positivity in childhood onset Graves' disease treated with propylthiouracil. J Clin Endocrinol Metab 2000;85:4270-3.

7 Merkel PA. Drugs associated with vasculitis. Curr Opin Rheumatol 1998; 10:45-50.

8 Levine JS, Branch W, Rauch J. The antiphospholipid syndrome. N Engl J Med 2002;346:752-63. 